\title{
Article
}

\section{The Pattern of Affective Responses to Dance-Based Group Exercise Differs According to Physical Fitness, as Measured by a Smartwatch}

\author{
Yujin Kim ${ }^{1}$, Jihye Kim ${ }^{2}$ and Minjung Woo ${ }^{2, *}$
}

check for updates

Citation: Kim, Y.; Kim, J.; Woo, M. The Pattern of Affective Responses to Dance-Based Group Exercise Differs According to Physical Fitness, as Measured by a Smartwatch. Appl. Sci. 2021, 11, 11540. https://doi.org/ 10.3390/app112311540

Academic Editor: Nyeonju Kang

Received: 4 November 2021

Accepted: 2 December 2021

Published: 6 December 2021

Publisher's Note: MDPI stays neutral with regard to jurisdictional claims in published maps and institutional affiliations.

Copyright: (c) 2021 by the authors. Licensee MDPI, Basel, Switzerland. This article is an open access article distributed under the terms and conditions of the Creative Commons Attribution (CC BY) license (https:/ / creativecommons.org/licenses/by/ $4.0 /)$.
1 Department of Physical Education, Sejong University, Seoul 05006, Korea; ykim@sju.ac.kr

2 School of Exercise and Sport Science, University of Ulsan, Ulsan 44610, Korea; rlawlgp1107@naver.com

* Correspondence: mjwoo@ulsan.ac.kr

\begin{abstract}
The present study investigated the effect of a dance-based aerobic exercise, on the affective experiences of participants with different fitness levels. Thirty-two college students were enrolled in the same dance fitness course, tested using a physical fitness test (the National Fitness Project 100) and grouped accordingly to 15 sports majors (high-fit group) and 17 non-sports majors (low-fit group). Together, they participated in a single-session dance fitness program using 11 basic dance steps incorporated in Zumba rhythms of merengue and reggaeton for $47 \mathrm{~min}$ including warm-up and cool-down. Pre- and post-exercise affects were measured using the PANAS- $X$ transmitted to each participant's smartphone. During exercise, participants' heart rate (HR) and their responses to the felt arousal scale (FAS) and the feeling scale (FS) by exercise section were measured using tailor-made applications on a smartwatch. Results showed that the intensity of exercise for the same exercise program was lower in the high-fit group than in the low-fit group, as evidenced by \%HRmax. In addition, the pattern of affective change throughout the exercise sections was different according to the groups' fitness levels, while the affective improvement was greater in the high-fit group. This study confirmed that physical fitness is a major variable influencing the relationship between exercise and affect.
\end{abstract}

Keywords: affect; physical fitness; dance-based group exercise; wearable technology; smartwatch

\section{Introduction}

It has been reported that regular exercise has mental health benefits such as reducing depressive symptoms and improving mood and self-esteem [1]. In particular, aerobic exercise is mainly conducive to stress relief and emotional stability, contributing to mental health [2]. In addition, because aerobic exercise has such a powerful effect on depression, some have even referred to it as an antidepressant with no side effects [3].

The psychological benefits of exercise participation vary depending on exercise intensity, duration, and type [4,5]. For example, exercise intensity is a major influencing factor of affect, in that exercise at a moderate to a vigorous intensity below the ventilatory threshold (VT) or lactate threshold (LT) is known to induce positive affective changes [6-9]. One can usually maintain a pleasant affect until the VT, but a sudden shift occurs from a pleasant to unpleasant affect upon exceeding the VT. During exercise at the VT level, the affective experience of individuals gradually declines in the direction of displeasure, which bounces back to pleasure once the exercise completes [6-10]. As such, the affective experience during exercise varies according to the intensity of exercise.

Despite a positive affect restored after exercise, the discomfort experienced during the prior exercise can influence exercise adherence or withdrawal in the future [11]. Therefore, the affective point of view does not recommend immediately exposing beginners to exercise at a high intensity because it is likely to induce negative affective responses during exercise. Furthermore, even with the same exercise program, individuals can feel the intensity of 
exercise differently depending on their physical fitness level. For example, a moderateintensity exercise of $60 \%$ of HRmax for the high-fit group may be equivalent to a vigorousintensity exercise of more than $90 \%$ of HRmax for the low-physical group [12]. However, little research has investigated how the affective benefit of exercise varies depending on the fitness status of an exerciser.

In a study examining the affective changes during vigorous-intensity exercise in association with exercise intensity preference, the group that preferred low-intensity exercise exhibited gradually increasing displeasure three minutes after the start of the exercise to the end of the exercise $(15 \mathrm{~min})$. The affect returned to the pre-exercise level after the exercise. On the other hand, the group that preferred high-intensity exercise maintained the initial level of affect throughout the exercise, and the pleasure increased after the exercise [13]. These results suggest that the impact of exercise on our affective experience can vary significantly according to one's preference for high-intensity exercise. Considering that high-fit individuals have a higher preference for vigorous-intensity exercise than low-fit individuals [14], it can be predicted that there will be differences in affective changes during exercise depending on physical fitness. Therefore, it is of importance to investigate how physical fitness mediates affective experience associated with exercise.

Another important variable that comes into play between exercise and affect is the dynamics experienced when exercising with others. For example, according to the social interaction hypothesis [15] or the psychosocial hypothesis [16], the interaction between people participating in exercise or positive attention from those exercising together is a key factor in affective improvement we receive from exercise. Furthermore, the degree to which exercise increases endorphins is greater when a group is together than alone [17]. A rhythmic exercise accompanied by music may add benefit to the mood-lifting effect $[18,19]$. In this regard, considering the dynamic factors involved in the effect of exercise on affect, researchers should conduct studies on the exercise-affect relationship in a real-life exercise field rather than in a laboratory setting.

To date, the existing research tools have not been capable of accurately measuring the constantly changing affect and exercise intensity (heart rates) at the same time, particularly for a large group of people in the exercise field. However, with the recent development of the Internet of things (IoT), several wearable devices have been introduced [20]. Of these devices, the smartwatch enables us to send, respond, and collect questionnaires and measure the heart rate required for identifying exercise intensity and automatically store all the data in the cloud. Smartwatches generally use photoplethysmographic (PPG) signals to measure heart rate [21]. Prior studies verified the accuracy and reliability of heart rate measured with smartwatches in varying conditions (e.g., rest, walking, cycling, dancing) [22]. In addition, it is possible to control multiple smartwatches simultaneously, transmit and store data automatically via an application developed to suit the purpose. Thus, wearable devices and mobile apps have made field research possible beyond existing research tools.

Zumba fitness is one of the representative dance-based sports that have recently dominated the group exercise market. Zumba fitness is a workout program that blends energetic Latin music with basic dance steps in a fitness program that encourages participants to enjoy aerobic exercise and have fun, as indicated by its official motto, "Ditch the workout. Join the party" [23]. The exercise intensity of Zumba is known to be $66 \pm 10.5 \%$ of the average VO2max, consuming $369 \pm 108 \mathrm{Kcal}$ for $40 \mathrm{~min}$ [24], which meets the American College of Sports Medicine (ACSM)'s recommended dose to maintain a healthy weight [25]. Research evidence suggests that Zumba effectively improves aerobic fitness [26] and weight loss [27]. In addition, participation in Zumba fitness enhances positive affect. In a recent study, Lee et al. [28] investigated the affective changes during Zumba fitness as a function of exercise intensity. They found that 45 -min low-intensity and moderate-intensity Zumba programs both affected improving effect during exercise, with greater positive affect observed in moderate intensity relative to low intensity. Furthermore, Zumba fitness improves quality of life [29] and alleviate depressive symptoms [30]. 
Therefore, this study uses a smartwatch in a real-life exercise setting to investigate the effect of a dance-based aerobic group class on the affective experiences of participants with different fitness levels.

\section{Materials and Methods}

\subsection{Participants}

Thirty-two sports major $(n=15,23.33 \pm 1.88$ years old) and non-major $(n=17$, $20.59 \pm 1.67$ years old) college students were enrolled in the same dance fitness course and voluntarily participated in the present study. A physical fitness test was conducted on each participant. Based on these results, the participants were classified according to physical fitness into high-fit and low-fit groups (Table 1). Participants were instructed to refrain from alcohol intake $24 \mathrm{~h}$ before the experiment and excessive exercise $72 \mathrm{~h}$ before the experiment. The authors' Institutional Review Board (1040968-A-2020-020) approved the research protocol for this study. The sample size required for this research design was estimated using the $\mathrm{G}^{*}$ power calculator (version 3.1.9.4; Düsseldorf University, Düsseldorf, Germany) [31]. The sample size was estimated based on a significance level of 0.05 , the statistical power of 0.90 , and the effect size found in a previous study investigating affective changes during a group dance program $\left(\eta^{2}=0.497\right)$. Based on these criteria, the estimated sample size was 18 people, meaning that nine or more participants in each high-fit and lowfit group would yield a statistical power above 0.90 . Therefore, the number of participants in this study $(n=32)$ is expected to have sufficient statistical power.

Table 1. Demographic information of participants.

\begin{tabular}{ccccc}
\hline \multirow{2}{*}{ Item } & \multicolumn{2}{c}{ High-Fit Group } & \multicolumn{2}{c}{ Low-Fit Group } \\
\cline { 2 - 5 } & $\boldsymbol{M}$ & $\boldsymbol{S D}$ & $\boldsymbol{M}$ & $\boldsymbol{S D}$ \\
\hline Age (yrs.) & 23.33 & 1.88 & 20.59 & 1.67 \\
BMI (kg/m $\left.{ }^{2}\right)$ & 23.56 & 3.96 & 22.63 & 3.84 \\
Body fat (\%) & 25.09 & 6.94 & 31.87 & 7.94 \\
Fitness (Z-score) & 0.36 & 0.69 & -0.52 & 0.56 \\
\hline
\end{tabular}

\subsection{Materials}

\subsubsection{Physical Fitness Measures}

To measure the participants' physical fitness, the National Fitness Project 100 developed by the Korean Ministry of Culture, Sports and Tourism was used [32,33]. This measure consists of six categories: muscular strength (grip strength), muscular endurance (cross sit-ups), cardiorespiratory endurance (20 m shuttle run), flexibility (seated forward bend), agility ( $4 \times 10 \mathrm{~m}$ shuttle run), and power (standing long jump). The fitness outcome values for all six categories were standardized into $Z$ scores to minimize the influence of age and gender variables using the mean and standard deviation of the population (men and women in the early $20 \mathrm{~s}$ ) provided by the Ministry of Culture, Sports and Tourism [33] (Formula (1)).

$$
\mathrm{Zscore}_{i j}=\frac{Y_{i j}-\overline{Y_{i}}}{S_{Y}}
$$

(i: participants, $j$ : fitness category, $Y$ : fitness score, $\bar{Y}$ : population mean, $S_{Y}$ : population SD).

After obtaining the total fitness scores by averaging the standardized $Z$ scores in all fitness categories for individual participants, an independent sample t-test was performed to verify the difference in the physical fitness measure between the groups. Results showed that the overall fitness score of the high-fit group $(Z=0.36)$ was higher than that of the low-fit group $(Z=-0.52)(t=3.96, p<0.001)$ (Table 1$)$.

\subsubsection{Smartphone}

An android-based smartphone (Galaxy Note 9, Samsung, Seoul, Korea) was used where we installed a tailor-made app for remote control of the smartwatches and moni- 
toring the measurement status. For data collection on arousal and affective responses of participants during Zumba fitness, all the smartwatches worn by the participants were controlled simultaneously by this single central smartphone.

\subsubsection{Smartwatch}

TicWatch E (Mobvoi, Beijing, China) was used, an Android Wear OS 2.0-based smartwatch capable of running our tailor-made app and equipped with a GPS, heart rate (HR) sensor, proximity sensor, balance sensor, and accelerometer. TicWatch $\mathrm{E}$ has reliable accuracy, showing more than 95\% correlation and agreement than the HR measured by Polar (wireless HR monitor, POLAR) during walking, dancing, and cycling as well as at rest [22]. Because the smartwatch's adhesion to the wearer's skin can impair HR measurement accuracy [34]. Kinesiology tape was applied around the watch to reduce movement and loss of light from the sensor during workouts. The watch was securely fastened to the participant's left wrist, approximately $2-3 \mathrm{~cm}$ below the ulnar styloid process. All participants wore smartwatches, measuring heart rate, arousal, and mood during Zumba fitness exercise.

\subsubsection{Application Development and Smartwatch Control}

In collaboration with a software engineering expert, an application was developed to collect HR and affective data and another one to control smartwatches. The app installed on the smartphone for the measurement of HR and affect was to activate the HR sensor of smartwatch to initiate HR measurement and to collect data via participants' affective self-reports, which was designed to be stored automatically on Google Cloud. A watch control app was also developed on the Galaxy S9 with Android Pie (version 9.0) to ensure that the measurement app operated effectively on the smartwatch. The watch control app comprised four modes: watch connection, HR measurement, affect measurement, and data management. In the "watch connection" mode, the connection status of up to 30 smartwatches was monitored. The "HR measurement" mode was used to initialize, start, pause, or stop the smartwatch HR sensor. In the "affect measurement" mode, a vibration alarm was sent to the participants on their smartwatches, which cued to report current feelings and arousal states by selecting responses on the watch's touch screen. The HR and affective data were stored in the Google Cloud for real-time monitoring (Figure 1). All data acquired during the experiment were freely accessible to be downloaded or deleted via the "data management mode".

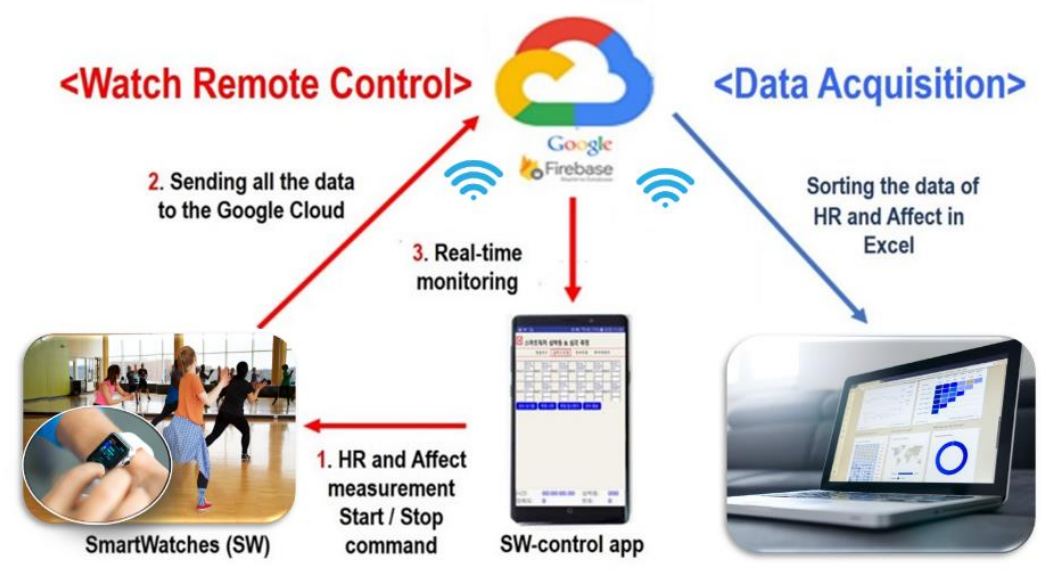

Figure 1. Description of smartwatch control and data acquisition processes.

\subsection{Affective Measures}

2.3.1. Positive and Negative Affect Schedule-Expanded Form (PANAS-X)

To measure the affective changes before and after Zumba fitness, the Korean version of the Positive Affect and Negative Affect Schedule-expanded form (PANAS-X) was used [35]. The PANAS- $X$ is a five-point 20-item scale comprising 10 positive and 10 negative items (i.e., 1 = not at all, $2=$ a little, $3=$ moderately, $4=$ quite a bit, $5=$ very much). 
With an internal consistency coefficient of 0.84 , the PANAS-X scale is relatively reliable, and the positive and negative affect scales are independent. We created the online version of the 20-item PANAS-X with Google Forms and transmitted the link to the smartphones of study participants for a response. Their responses were stored automatically on Google Drive. In the present study, the Cronbach's alpha coefficients were 0.61 for the PA and 0.66 for the NA scales.

\subsubsection{Two-Dimensional Circumplex Model of Affect}

Russell 's [36] two-dimensional circumplex was used to track the affective changes throughout the entire Zumba Fitness program. As presented in Figure 2, the model consists of four quadrants, each of which represents arousal level (activation-deactivation continuum) and valence (pleasure-displeasure continuum). In the two-dimensional circumplex model, the affective state can be identified by the point of intersection, with the $x$ and $y$ axes signifying valence (pleasure, displeasure) and arousal (activation-deactivation continuum), respectively (Figure 2). Russell [37] defined this intersecting point as a core affect reflecting valence and activation. The valence dimension was assessed by the feeling scale (FS), while the arousal dimension reflected the felt arousal scale (FAS).

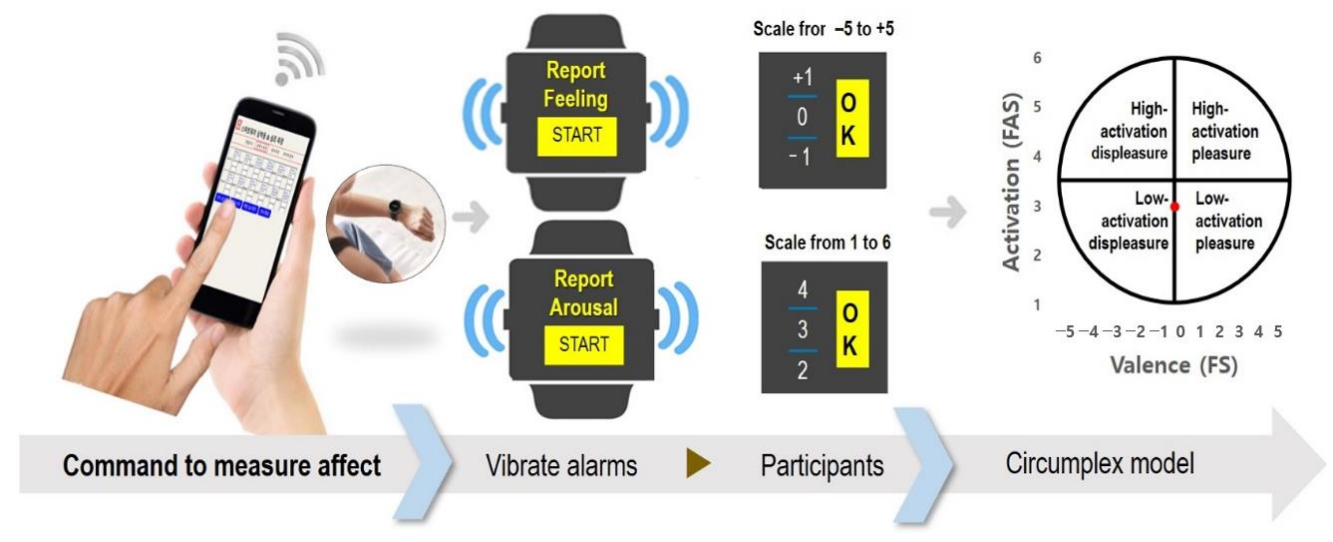

Figure 2. The two-dimensional circumplex model of affect and the smartwatch display for measuring affect and arousal.

This model consists of four quadrants: The first quadrant describes activation-pleasure (excited, energized, and passionate), while the second quadrant reflects activation-displeasure (anxious, nervous, and stressed). The third quadrant represents deactivation-displeasure (bored, tired, and depressed), and the fourth quadrant represents deactivation-pleasure (relaxed and calm) [36]. The smartwatch interface was used to implement the feeling scale (FS) and the felt arousal scale (FAS) to simultaneously evaluate affect in multiple participants engaging in the group exercise concurrently.

\subsubsection{The Feeling Scale (FS)}

A single item 11-point bipolar FS (pleasure/displeasure) devised by Hardy and Rejeski [38] was used to assess during exercise affective responses on the valence dimension ( $x$-axis) of the two-dimensional circumplex model of affect. When participants received a vibration alarm sent from the central watch control app with the message "report Feeling" on the smartwatch screen, the app guided participants to press the start button and scroll up or down the touch screen to choose a number from -5 to +5 corresponding to their current feelings (Figure 2). The Cronbach's alpha coefficient of the FS in the present study was 0.89 .

\subsubsection{The Felt Arousal Scale (FAS)}

The FAS devised by Svebak and Murgatroyd [39] was used to evaluate the level of arousal on the arousal dimension (y-axis) of the two-dimensional circumplex model. This 
single-item six-point scale ranging from 1 (low arousal or deactivation) to 6 (high arousal or activation) was used to assess arousal levels during exercise. When the "report arousal" screen appeared on participants' smartwatches with a vibration alarm, the participants chose a number from 1 to 6 corresponding to their current activation status. Then, they sent the response by pressing the OK button (Figure 2). The Cronbach's alpha coefficient of the FAS in the present study was 0.73 .

\subsection{Dance Fitness Program}

The dance fitness program was developed using 11 basic dance steps incorporated in Zumba rhythms of merengue and reggaeton, consisting of 8-min warp-up, 8-min basic steps, 8-min merengue, 8-min reggaeton, 15-min cool-down with stretching, all totaling 47 -min of exercise. Table 2 presents the details of the steps, music, and beat per minute used in the program.

Table 2. Dance fitness program.

\begin{tabular}{|c|c|c|c|c|c|c|}
\hline Rhythm & \multicolumn{4}{|c|}{ Basic Steps } & Music & bpm \\
\hline Aerobics & \multicolumn{4}{|c|}{$\begin{array}{l}\text { March, step touch, V-step, lunge, heel-jack, } \\
\text { knee up, grapevine, mambo, back-up, box, tap }\end{array}$} & Top Ten 24 \#1 & 130 \\
\hline Merengue & March & 2 Step & 6 Step & $\begin{array}{l}\text { Beto } \\
\text { Shuffle }\end{array}$ & $\begin{array}{c}\text { Basic } 1 \text { review music } \\
\text { Fiesta }\end{array}$ & $\begin{array}{l}124 \\
124\end{array}$ \\
\hline Reggaeton & Stomp & Knee-lift & Destroza & $\begin{array}{l}\text { Step } \\
\text { bounce }\end{array}$ & $\begin{array}{l}\text { Basic } 1 \text { review music } \\
\text { Toma reggaeton }\end{array}$ & $\begin{array}{l}94 \\
96\end{array}$ \\
\hline
\end{tabular}

\subsection{Procedures}

Participants who signed the informed consent visited the National Fitness 100 Center to have their physical fitness measured, required for completion $72 \mathrm{~h}$ before the experiment. When participants arrived, the smartwatch firmly fitted on their left wrist, 2 to $3 \mathrm{~cm}$ below the ulnar styloid process. Then, they were instructed to sit and rest on a yoga mat. The experimenter then initiated the watch control app on the smartphone, checked the connection of all watches, and tested if the alarm transmission and HR measurement worked properly. After explaining the experimental procedure, we transmitted the 20-item PANAS- $X$ to each participant's smartphone to be answered before beginning the exercise. To begin the Zumba fitness program, participants stood with enough space between them and faced the mirror. At the start of the session, the experimenter activated the HR sensors of participants' smartwatches by pressing the start button of the HR measurement mode of the watch control app. At the end of each exercise section (warm-up, basic steps, merengue, reggaeton), the experimenter hit the stop button of the watch control app to finish HR measurement and transmitted an alarm in the affect measurement mode. Once all the participants completed responding to the FS and FAS scales by touching the smartwatch screen, the watch control app confirmed the completed responses, and the group proceeded to the next exercise section.

As shown in Figure 3, the PANAS-X pre-and post-exercise and the HR during exercise (warm-up, basic steps, merengue, reggaeton) were measured. The FS and FAS were measured six times (pre-exercise after PANAS-X, $8 \mathrm{~min}, 17 \mathrm{~min}, 26 \mathrm{~min}, 35 \mathrm{~min}$, and postexercise before PANAS-X). The time required for answering the FS and FAS in between sections was 20 to $40 \mathrm{~s}$. When the Zumba program was over with reggaeton, followed by a cool-down, participants responded to the post-exercise FS and FAS while resting on the yoga mat. Then, the PANAS-X link was sent to the participants' smartphones to respond. After completing the response and returning the watch to the experimenter, participants returned home. 


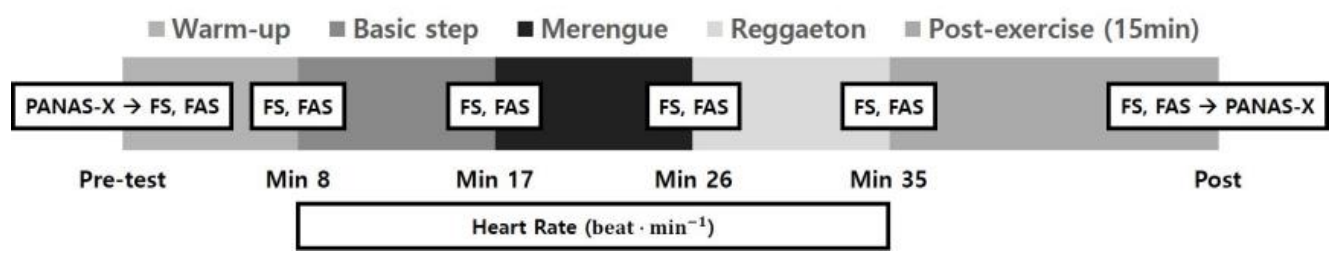

Figure 3. The measurement of HR, affect, and arousal before, during, and after exercise.

\subsection{Data Collection and Processing}

The HR measured during exercise by section (warm-up: exercise start to $8 \mathrm{~min}$, basic step: $8-17 \mathrm{~min}$, merengue: $17-26 \mathrm{~min}$, reggaeton: $26 \mathrm{~min}$ to completion) were averaged to verify whether a difference existed in the exercise intensity of the dance exercise program between the high-fit and low-fit groups. Then, the relative exercise intensity of the participants was calculated using the HR data and the HRmax estimation formula $(\mathrm{HRmax}=220$-age) $[25,40]$. Finally, the exercise intensity was calculated by dividing the HRmax by the during-exercise HR, multiplied by 100 [41].

\subsection{Statistical Analysis}

All statistical analyses were performed using the SPSS Version 25.0 for Windows. Mauchly's sphericity test was performed on all dependent variables and adjusted the degree of freedom of ANOVA through Greenhouse-Geisser for variables that did not meet the sphericity assumption. The level of statistical significance of ANOVAs was set to 0.05 , and the significance level for HR and exercise intensity was 0.0031 for post-hoc analysis. The significance levels for the PANAS-X and the FS and FAS were 0.0125 and 0.0014 , respectively. An analysis of variance (ANOVA) was performed to investigate the interaction of HR, exercise intensity, PANAS-X, FS, and FAS as a function of group and time. To examine differences in HR and exercise intensity, separate 2 (group: high-fit vs. low-fit) $\times 4$ (time: warm-up, basic steps, merengue, reggaeton) two-way repeatedmeasures ANOVAs were performed. In addition, the positive and negative affect of the PANAS- $X$ was analyzed by a 2 (group: high-fit vs. low-fit) $\times 2$ (time: pre- vs. post-exercise) two-way repeated-measures ANOVA. Finally, separate 2 (group: high-fit vs. low-fit) $\times 6$ (time: pre-exercise, $8 \mathrm{~min}, 17 \mathrm{~min}, 26 \mathrm{~min}, 35 \mathrm{~min}$, post-exercise) two-way repeatedmeasures ANOVAs were performed using FS and FAS as dependent variables. In case of an interaction effect between the group and time, a post-hoc analysis was performed. The significance level was set through Bonferroni adjustment for all post-hoc analyses.

\section{Results}

\subsection{HR and Exercise Intensity (\%HRmax)}

In the analysis of HR by measurement time, significant group $(F(1,30)=20.646$, $p<0.001, d=1.18$, CI $[0.79,2.40])$ and time $(F(2.2,30)=12.936, p<0.001, d=1.66$, $\mathrm{CI}[0.88,1.77])$ main effects emerged. The HR of the high-fit group during exercise was lower than that of the low-fit group $(p<0.001)$ (Figure 4$)$.

As a result of post-hoc analysis of the main effect of time, the HR during basic steps $(p=0.002)$ and reggaeton exercise $(p<0.001)$ was higher than the HR during the warmup exercise, and the HR during reggaeton exercise was higher than the HR during the merengue exercise $(p<0.001)$. In the analysis of exercise intensity, calculated by \%HRmax, significant main effects of group $(F(1,30)=17.522, p<0.001, d=1.53, \mathrm{CI}[10.05,2.09])$ and time $(F(2.197,30)=12.882, p<0.001, d=1.31, \mathrm{CI}[0.88,1.76])$ emerged, with the high-fit group exhibiting lower exercise intensity compared to the low-fit group $(p<0.001)$. The post-hoc analysis on the main effect of time revealed that the exercise intensity of the basic steps $(p=0.002)$ and reggaeton exercise $(p<0.001)$ was higher than that of the warmup exercise, and the exercise intensity of reggaeton was higher than that of merengue $(p<0.001)$. However, the interaction for group $\times$ time was not significant in HR and exercise intensity. 

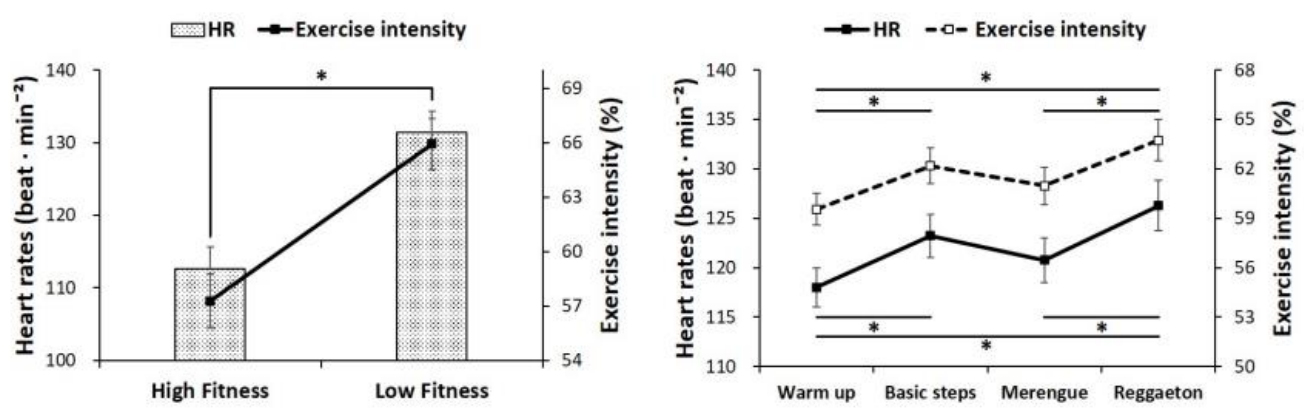

Figure 4. Differences in heart rates and the exercise intensity between groups (left) and times (right). Note: all the error bars represent standard errors. In the difference between times (left), the significant value adjusted by Bonferroni correlation is $p<0.0031$. * represents a significant difference.

\subsection{PANAS-X}

\subsubsection{Positive Affect}

A significant main effect of group emerged $(F(1,30)=5.585, p=0.025, d=0.86$, $\mathrm{CI}[0.59,1.18])$, with the high-fit group exhibiting higher positive affect relative to the lowfit group. However, the main effect on time was not significant. As a result of ANOVA before and after exercise between groups, we found a significant interaction effect between groups $\times$ period $(F(1,30)=8.261, p=0.007, d=1.05, \mathrm{CI}[0.72,1.44])$. The post-hoc analysis revealed no significant group difference in pre-exercise positive affect. Meanwhile, the post-exercise positive affect was significantly higher in the high-fit group compared to the low-fit group $(p=0.003)$ (Figure 5). However, no significant difference was observed in positive affect between pre- and post-exercise within each group.
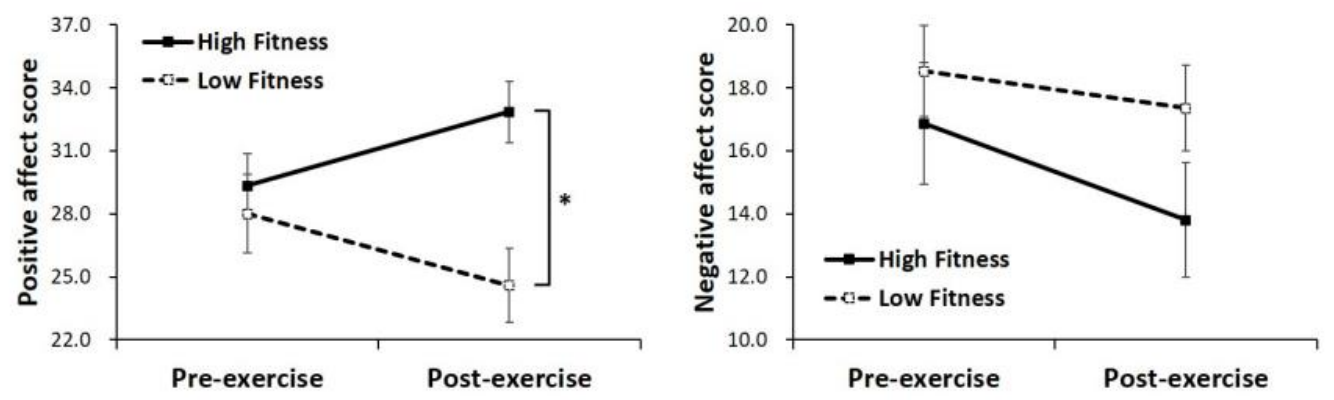

Figure 5. Differences in positive (left) and negative (right) affective responses between groups and times. Note: solid lines represent the high fitness group, and dash lines represent the low fitness group. All error bars speak of standard errors. $p<0.0125$. ${ }^{*}$ represents a significant difference.

\subsubsection{Negative Affect}

The analysis of pre- and post-exercise negative affect between groups yielded no significant main effect or interaction effect (Figure 5).

\subsection{The Feeling Scale and Felt Arousal Scale \\ 3.3.1. The Feeling Scale}

In the analysis of the FS, there was a significant main effect of time on the FS scores $(F(3.3,30)=14.306, p<0.001, d=1.38, \mathrm{CI}[0.91,1.83])$, while the main effect of group did not reach significance. In addition, a significant group $\times$ time interaction effect $(F(3.3,30)=3.192, p=0.023, d=0.65, \mathrm{CI}[0.43,0.86])$ emerged (Figure 6). As a result of the post-hoc analysis, the high-fit group showed significantly higher FS scores at $26 \mathrm{~min}$ and $35 \mathrm{~min}$ than pre-exercise $(p<0.001389)$. In contrast, the low-fit group exhibited higher FS scores after exercise than pre-exercise, $17 \mathrm{~min}, 26 \mathrm{~min}$, and $35 \mathrm{~min}(p<0.001389)$. However, no significant difference emerged between the groups as a function of time. 


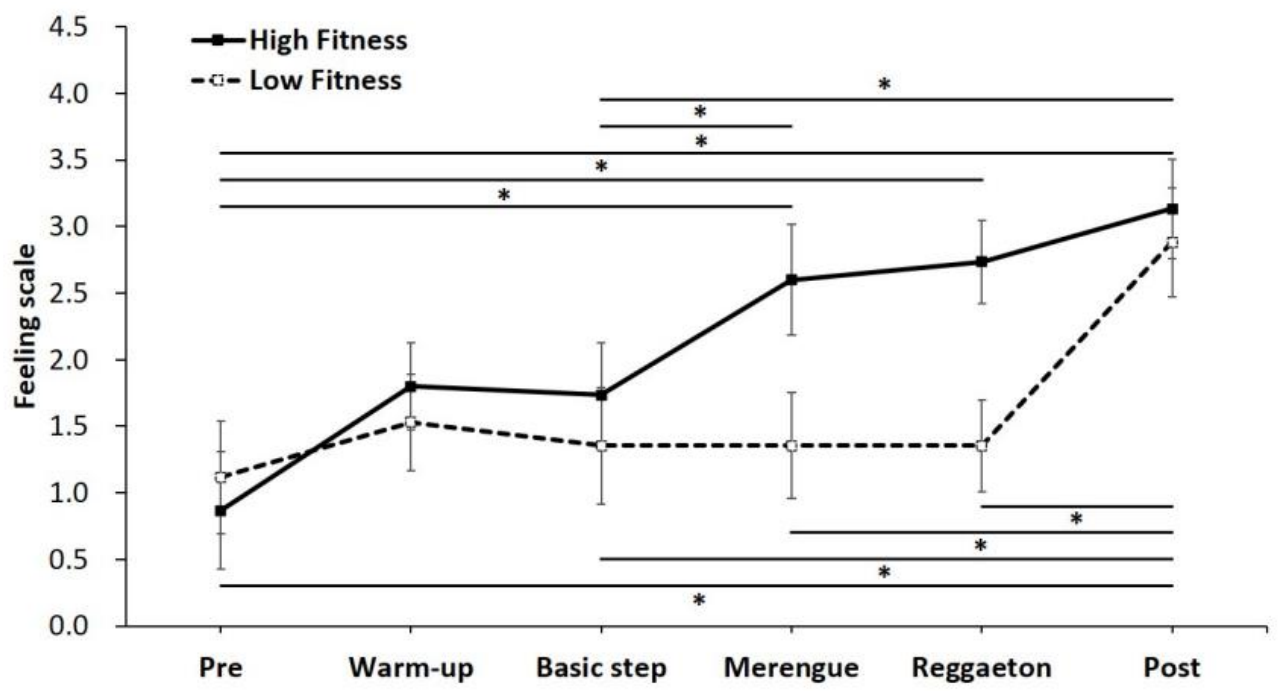

Figure 6. Differences in the feeling scale between groups and times. Note: solid lines represent the high fitness group, and the dashed line represents the low fitness group. All error bars speak of standard errors. $p<0.0014 .{ }^{*}$ represents a significant difference.

\subsubsection{The Felt Arousal Scale}

In the analysis of the FAS scores, a significant main effect of time emerged $(F(3.3,30)=9.916$, $p<0.001, d=1.15, \mathrm{CI}[0.76,1.52])$, but the main effect of group was not significant. A group $\times$ time interaction effect was also significant $(F(3.1,30)=2.968, p=0.034, d=0.63$, $\mathrm{CI}[0.42,0.83]$ ) (Figure 7). The post hoc analysis revealed that the FAS score of the high-fit group was higher at $26 \mathrm{~min}$ compared to pre-exercise, $8 \mathrm{~min}$, and $17 \mathrm{~min}$, and the score was also higher at $35 \mathrm{~min}$ compared to pre-exercise and $17 \mathrm{~min}(p<0.001389)$. However, the low-fit group exhibited no significant difference in the FAS score depending on time, with no group $\times$ time interaction.

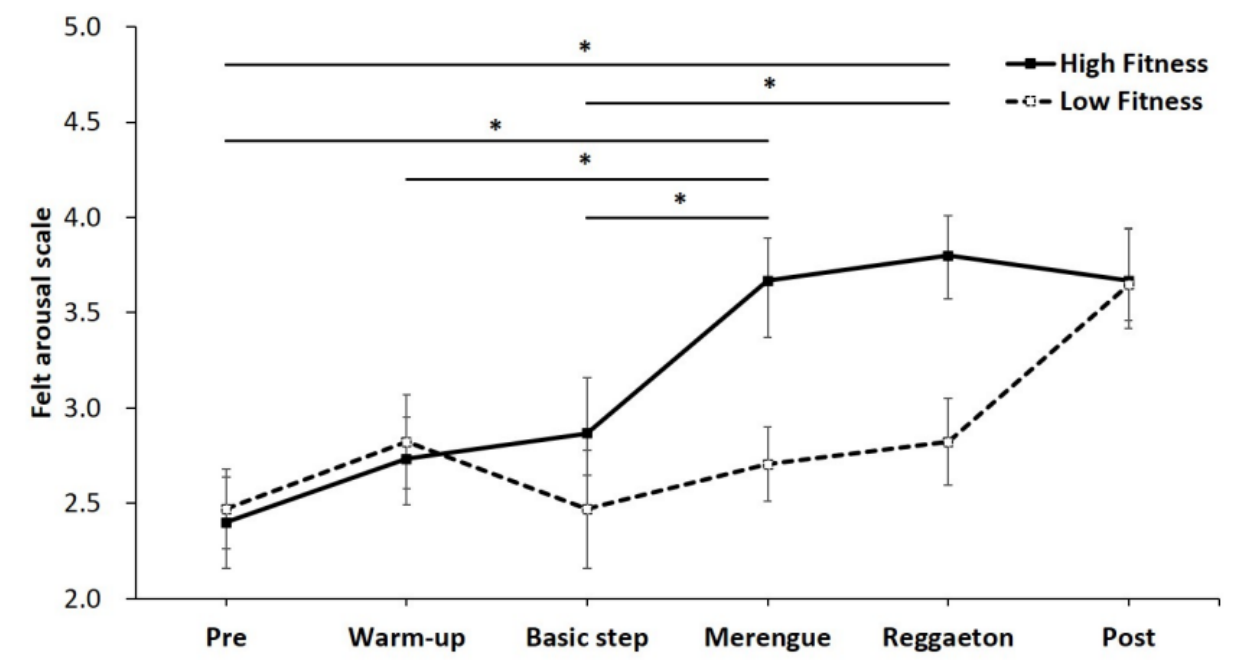

Figure 7. Differences in the felt arousal scale between groups and times. Note: solid lines represent the high fitness group, and the dashed line represents the low fitness group. All error bars speak of standard errors, $p<0.0014 .{ }^{*}$ represents a significant difference.

\subsection{The Two-Dimensional Circumplex Model}

According to time by group, the affective changes are depicted in a two-dimensional circumplex model of valence and activation in Figure 8. For example, the core affect of the low-fit group remained in the fourth quadrant (deactivation-pleasure) without significant change from pre-exercise at $35 \mathrm{~min}$. It then moved to the first quadrant (activation-pleasure) 
after exercise. On the other hand, the core affect of the high-fit group gradually improved within the fourth quadrant (deactivation-pleasure) from pre-exercise. Finally, it advanced to the first quadrant (activation-pleasure) after $26 \mathrm{~min}$ (merengue), which participants maintained until exercise completion.

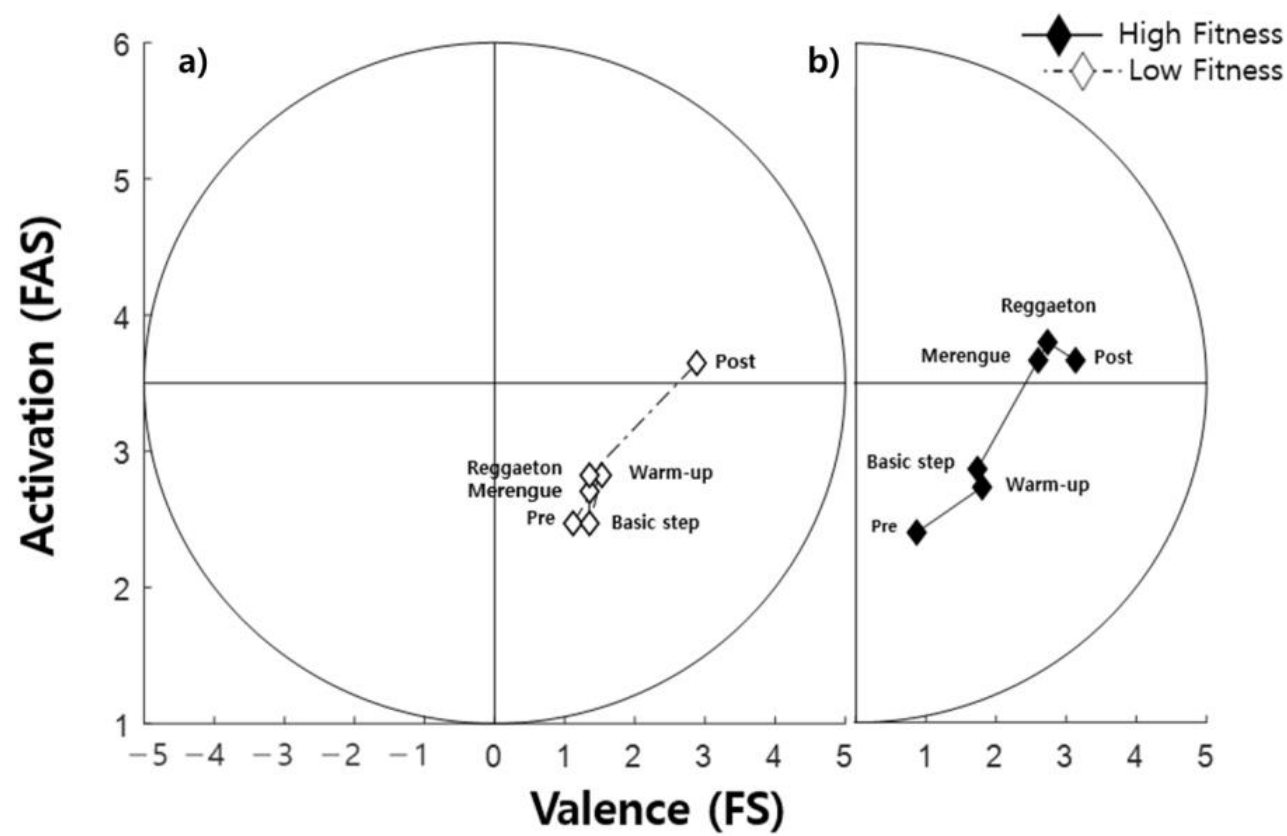

Figure 8. Two-dimensional circumplex model of affect by the level of fitness, (a) high fitness and (b) low fitness. Note: the circumplex model is a method to observe a change of 'core' affect (valence + activation). Quadrant I represents activation-pleasure; Quadrant II represents activationdispleasure; Quadrant III represents deactivation-displeasure; Quadrant IV represents deactivationpleasure.

\section{Discussion}

This study used a smartwatch to investigate whether a dance-based aerobic group exercise affects participants' affective responses depending on their level of physical fitness. In the analysis of the difference in exercise intensity of Zumba fitness according to physical fitness, the high-fit and low-fit groups exhibited differences in HR and exercise intensity (\%HRmax) during $35 \mathrm{~min}$ of Zumba exercise from warm-up to just before cool-down. Specifically, HR during exercise was $109 \mathrm{bpm}$ with 57\% HRmax exercise intensity for the high-fit group and $130 \mathrm{bpm}$ with $66 \% \mathrm{HRmax}$ intensity for the low-fit group.

The ACSM [25] indicated that the relative intensity of aerobic exercise is $57-63 \%$ HRmax for low intensity and 64-76\% HRmax for moderate intensity. Therefore, the Zumba fitness program used in this study is of moderate intensity for the low-fit individuals and lowintensity for the high-fit participants. This study's Zumba exercise intensity supports the findings of Luettgen et al. [24] that the exercise intensity of Zumba for the general public is $66 \pm 10.5 \%$ of the average VO2max, which meets the ACSM criteria for maintaining a healthy weight. Furthermore, by tracking HR during exercise in real-time using a smartwatch, our study verified that exercise intensity differs depending on the performer's fitness level. Our study's Zumba fitness program consisted of a warm-up, basic dance steps, merengue, and reggaeton. In the comparison of exercise intensity according to the exercise section, we found that the exercise intensity of reggaeton was the highest compared to warm-up, basic steps, and merengue. This result is consistent with Lee et al. [28], who reported the highest HR during reggaeton exercise among the four Zumba rhythms: merengue, reggaeton, salsa, and cumbia. While merengue consists of up-down movements of the pelvis, reggaeton creates up-down motions of the entire lower body through flexion and extension, thus intensifying the exercise. In addition, since the reggaeton steps use 
two steps per $1 \mathrm{bpm}$ [42], it is possible to double up the speed of the movements up to $192 \mathrm{bpm}$ [43]. Therefore, to control the intensity of a Zumba fitness program within a limited time, instructors often control the speed of choreography by adding or subtracting the portion of reggaeton rhythm or breaking down the rhythm.

In the analysis of the change in affect (valence) and arousal (activation) by exercise section of the Zumba fitness program, we found an interesting difference between the high-fit and low-fit groups. There was no significant change in high-fit group's affect from pre-exercise, warm-up, and basic steps. However, the affect improved after the merengue, and participants maintained that affect throughout the rest of the exercise program. On the other hand, there were no significant changes in affect throughout the entire exercise section in the low-fit group, but the affect improved immediately after the cool-down. Furthermore, similar affective changes were observed in pre, mid, and post-exercise depending on fitness in arousal. In the high-fit group, arousal increased after merengue, and participants maintained this affect until the end. In contrast, the low-fit group showed no difference between pre and during-exercise arousal.

As shown in the two-dimensional circumplex model, which presents the changes in the core affect (valence + activation) (Figure 8), the core affect of the high-fit group increased from the beginning. It proceeded to the first quadrant after merengue, and the participants maintained the affect until the end of the exercise. The low-fit group maintained the core affect without significant change in the fourth quadrant, including their pre-exercise state and throughout the entire section. They then moved to the first quadrant immediately after exercise, showing increased positive affect and arousal. These findings suggest that despite the shared feelings of elevated mood and arousal individuals feel after exercise, the affective experience during exercise may differ depending on participants' fitness level. In addition, high-fit individuals seem to experience a greater increase in affect throughout the exercise than low-fit individuals.

For quick and repeated measurement of changes in affect and arousal in real-time during exercise, a single item 11-point Likert scale $(-5$ to +5$)$ was used. In doing so, we only assessed the affective experience during exercise within the extent of high and low levels of valence (displeasure to pleasure) and arousal (deactivation to activation). To supplement this, the participants were asked to answer a 20-item PANAS-X to measure positive and negative affect before and after exercise. The results of the PANAS-X pre- and post-exercise and the results of affect (valence) measured by the FS pre-, during-, and post-exercise were not consistent. Figure 5 shows that the high-fit and low-fit groups did not differ in positive affect before exercise. However, the positive affect after exercise was higher in the high-fit group relative to the low-fit group.

Despite no statistical difference between groups as a function of time (pre and postexercise), positive affect increased after exercise in the high-fit group and decreased in the low-fit group. This tendency made the difference in positive affect between groups significant after exercise. However, we found no significant difference in negative affect according to the fitness level or time in this study, which suggests that participation in a group dance program such as Zumba fitness may not lead to meaningful changes in negative affect. These results are consistent with the study of Lee et al. [28], where negative affect measured before and after a Zumba fitness program was not different regardless of exercise intensity. This finding implies that improved positive affect rather than decreased negative affect provides the affective benefit in dance-based exercise.

Interestingly, the results of this study regarding pre and post-exercise affect are somewhat contradictory to previous studies that investigated affective changes following exercise. The existing studies primarily focused on exploring affective changes according to exercise intensity. Many of them reported that moderate-intensity exercise could expect a greater affective improvement than exercises at a too low or high intensity [44]. In a recent study by Lee et al. [28] which investigated affective changes before, during, and after exercise of Zumba fitness depending on exercise intensity, moderate-intensity $(72.19 \%$ HRmax) exercise led to a greater increase in positive affect than low-intensity $(62.48 \% \mathrm{HRmax})$ 
exercise. Lee et al. also found that affect and arousal during exercise at moderate intensity gradually increased. In contrast, low-intensity exercise only led to a temporary increase in positive affect at the beginning of the exercise, without additional improvement during and after the exercise. Therefore, the researchers suggested that the affective benefit of exercise is greater when performed at moderate intensity than at low intensity. Our results contradict previous studies: we found that low-intensity (i.e., 57\% HRmax in high-fit group) exercise provides more significant affective benefit than moderate-intensity (i.e., $66 \%$ HRmax in low-fit group) exercise. However, the characteristics of the physical fitness variable need to be considered which influence the exercise-affect relationship.

Physical fitness is about moving the body to carry out a healthy and active daily life, which is the foundation of normal life [45]. Regular exercise enhances fitness [11]. Rezazadeh and Talebi [46] investigated how physical fitness mediates affective responses in firefighters and reported a positive correlation between physical fitness and affect and a negative correlation between BMI and affect. They concluded that since high-fit individuals have better emotional regulation ability, improving the fitness of firefighters would contribute to enhancing their work efficiency. Furthermore, individuals with higher aerobic fitness have a higher tolerance for exercise and a preference for high-intensity exercise [14]. The emotional regulation ability and exercise tolerance demonstrated in high-fit individuals are related to neurological and biological changes caused by long-term exercise participation.

In this study, although the high-fit group exercised at a lower intensity than the lowfit group, they exhibited a greater increase in positive affect, with affect and activation increasing faster during exercise and increasing gradually. Lin and Kuo [47] explained the benefits of exercise on brain function through monoamine connection. They indicated that regular moderate-intensity exercise improves adaptability and flexibility of the central nervous system (CNS). Exercise stimulates the monoaminergic systems (dopamine, noradrenaline, serotonin), thereby contributing to mental health without causing central fatigue. In addition, experiencing a repetitive increase in serotonin in the brain through regular exercise is known to act as an antidepressant [48]. Regular exercise participation also improves biological adaptation, which reduces physiological responses to stress [49]. Since exercise itself is a stressor, exposure to repeated exercise improves adaptability to stress and reduces the secretion of cortisol, a stress hormone, during exercise [50]. Having gained the ability to adapt to stress through regular exercise, high-fit individuals exhibit low levels of salivary cortisol secretion even in exercise-irrelevant stress situations [51,52]. As such, the neurophysiological changes due to long-term exercise participation may explain why the high-fit group in this study showed larger affective improvement than the low-fit group.

Physical fitness is a vital variable mediating affective experience during exercise. In this study, the pattern of affective change throughout the exercise sections was different according to the groups' fitness levels. The intensity of exercise for the same exercise program was lower in the high-fit group than in the low-fit group, while the affective improvement was greater in the high-fit group. This study confirmed that physical fitness is a major variable influencing the relationship between exercise and affect. However, we could not establish how fitness mediates affect through interaction with exercise intensity. Therefore, future studies should examine the interaction between fitness and exercise intensity to identify exercise conditions optimized for affective benefit.

In addition, developing a personalized exercise program that induces optimal affective improvement requires investigating the individual variables that mediate affective responses to exercise and the environmental variables that maximize the effect of exercise. In this regard, artificial neural networks or deep learning technology will become the ultimate goal of future exercise-affect research. This technology can aid in developing an algorithm capable of predicting and suggesting the best suitable exercise type, intensity, and duration based on individual (e.g., personality, physical fitness, genes, diseases) and environmental variables (e.g., exercise type, setting, interaction with others during exercise). 
Moreover, deep learning technology could maximize the overall mental health benefit of exercise.

Author Contributions: Conceptualization, M.W.; project administration, J.K. and M.W.; data analysis, J.K.; writing—original draft, M.W.; writing—translating and editing, Y.K.; manuscript preparation, Y.K.; supervision, M.W.; funding acquisition, M.W. All authors have read and agreed to the published version of the manuscript.

Funding: This work was supported by the Ministry of Education of the Republic of Korea and the National Research Foundation of Korea (NRF-2020S1A5A2A01045029).

Institutional Review Board Statement: The study was conducted according to the guidelines of the Declaration of Helsinki, and approved by the Institutional Review Board (or Ethics Committee) of University of Ulsan (1040968-A-2020-020).

Informed Consent Statement: Informed consent was obtained from all subjects involved in the study.

Conflicts of Interest: The authors declared no potential conflict of interest with respect to the research, authorship, and/or publication of this article.

\section{References}

1. White, K.; Kendrick, T.; Yardley, L. Change in self-esteem, self-efficacy and the mood dimensions of depression as potential mediators of the physical activity and depression relationship: Exploring the temporal relation of change. Ment. Health Phys. Act. 2009, 2, 44-52. [CrossRef]

2. Dimeo, F.; Bauer, M.; Varahram, I.; Proest, G.; Halter, U. Benefits from aerobic exercise in patients with major depression: A pilot study. Br. J. Sports Med. 2001, 35, 114-117. [CrossRef] [PubMed]

3. Sigwalt, A.R.; Budde, H.; Helmich, I.; Glaser, V.; Ghisoni, K.; Lanza, S.; Cadore, E.L.; Lhullier, F.L.; de Bem, A.F.; Hohl, A.; et al. Molecular aspects involved in swimming exercise training reducing anhedonia in a rat model of depression. Neuroscience 2011, 192, 661-674. [CrossRef]

4. Buckworth, J.; Dishman, R. Determinants of exercise and physical activity. In Exercise Psychology; Human Kinetics: Champaign, IL, USA, 2002; pp. 191-209.

5. Mun, C. The Effects of Preference Mode and Intensity of Exercise on Participants' Psychological and Physiological Responses. Korean J. Sport Psychol. 2011, 22, 149-169.

6. Bixby, W.R.; Lochbaum, M.R. Affect Responses to Acute Bouts of Aerobic Exercise in Fit and Unfit Participants: An Examination of Opponent-Process Theory. J. Sport Behav. 2006, 29, 111-125.

7. Ekkekakis, P.; Hall, E.E.; Petruzzello, S.J. The relationship between exercise intensity and affective responses demystified: To crack the 40-year-old nut, replace the 40-year-old nutcracker! Ann. Behav. Med. 2008, 35, 136-149. [CrossRef] [PubMed]

8. Kilpatrick, M.; Kraemer, R.; Bartholomew, J.; Acevedo, E.; Jarreau, D. Affective responses to exercise are dependent on intensity rather than total work. Med. Sci. Sports Exerc. 2007, 39, 1417-1422. [CrossRef]

9. Sheppard, K.E.; Parfitt, G. Acute Affective Responses to Prescribed and Self-Selected Exercise Intensities in Young Adolescent Boys and Girls. Pediatr. Exerc. Sci. 2008, 20, 129-141. [CrossRef] [PubMed]

10. Rose, E.A.; Parfitt, G. A quantitative analysis and qualitative explanation of the individual differences in affective responses to prescribed and self-selected exercise intensities. J. Sport Exerc. Psychol. 2007, 29, 281-309. [CrossRef]

11. Williams, D.M.; Rhodes, R.E.; Conner, M.T. (Eds.) Psychological hedonism, hedonic motivation, and health behavior. In Affective Determinants of Health Behavior; Oxford University Press: New York, NY, USA, 2018; pp. 204-234.

12. Physical Activity Guidelines Advisory Committee. Physical Activity Guidelines Advisory Committee Report; Department of Health and Human Services: Washington, DC, USA, 2008.

13. Box, A.G.; Petruzzello, S.J. Why do they do it? Differences in high-intensity exercise-affect between those with higher and lower intensity preference and tolerance. Psychol. Sport Exerc. 2020, 47, 101521. [CrossRef]

14. Schneider, M.; Graham, D. Personality, physical fitness, and affective response to exercise among adolescents. Med. Sci. Sports Exerc. 2009, 41, 947. [CrossRef]

15. Turner, J.H. A Theory of Social Interaction; Stanford University Press: Stanford, CA, USA, 1988.

16. Burke, P.J. Contemporary Social Psychological Theories; Stanford University Press: Stanford, CA, USA, 2020.

17. Mikkelsen, K.; Stojanovska, L.; Polenakovic, M.; Bosevski, M.; Apostolopoulos, V. Exercise and mental health. Maturitas 2017, 106, 48-56. [CrossRef]

18. Campion, M.; Levita, L. Enhancing positive affect and divergent thinking abilities: Play some music and dance. J. Posit. Psychol. 2014, 9, 137-145. [CrossRef]

19. Koch, S.; Kunz, T.; Lykou, S.; Cruz, R. Effects of dance movement therapy and dance on health-related psychological outcomes: A meta-analysis. Arts Psychother 2014, 41, 46-64. [CrossRef]

20. Thompson, M. Cultural Theory; Routledge: New York, NY, USA, 2018. 
21. Zhang, Z.; Pi, Z.; Liu, B. TROIKA: A general framework for heart rate monitoring using wrist-type photoplethysmographic signals during intensive physical exercise. IEEE. Trans. Biomed. 2015, 62, 522-531. [CrossRef]

22. Kim, J.; Lee, J.; Woo, J. Is heart rate measured by smartwatch during exercise reliable? Analysis of correlation and agreement between heart rates of Polar and smartwatch. J. Korea Converg. Soc. 2020, 11, 331-339.

23. Nieri, T.; Hughes, E. All about having fun: Women's experience of Zumba fitness. Sociol. Sport J. 2016, 33, 135-145. [CrossRef]

24. Luettgen, M.; Foster, C.; Doberstein, S.; Mikat, R.; Porcari, J. ZUMBA®: Is the "fitness-party" a good workout? J. Sci. Med. Sport 2012, 11, 357-358.

25. Riebe, D.; Ehrman, J.K.; Linguori, G.; Magal, M. (Eds.) American College of Sports Medicine's Guidelines for Exercise Testing and Prescription, 10th ed.; Kluwer: Philadelphia, PA, USA, 2017.

26. Donath, L.; Roth, R.; Hohn, Y.; Zahner, L.; Faude, O. The effects of Zumba training on cardiovascular and neuromuscular function in female college students. Eur. J. Sport Sci. 2014, 14, 569-577. [CrossRef]

27. Haghjoo, M.; Zar, A.; Hoseini, S.A. The Effect of 8 weeks Zumba Training on Women's Body Composition with Overweight. Pars Jahrom Univ. Med Sci. 2016, 14, 21-30. [CrossRef]

28. Lee, J.; Park, J.; Kim, Y.; Woo, M. Affective Change with Variations in Zumba Fitness Intensity as Measured by a Smartwatch. Percept. Mot. Skills 2021, 128, 2255-2278. [CrossRef]

29. Cugusi, L.; Wilson, B.; Serpe, R.; Medda, A.; Deidda, M.; Gabba, S.; Satta, G.; Chiappori, P.; Mercuro, G. Cardiovascular effects, body composition, quality of life and pain after a Zumba fitness program in Italian overweight women. J. Sports Med. Phys. Fit. 2016, 56, 328-335.

30. Norouzi, E.; Hosseini, F.; Vaezmosavi, M.; Gerber, M.; Puhse, U.; Brand, S. Zumba dancing and aerobic exercise can improve working memory, motor function, and depressive symptoms in female patients with Fibromyalgia. Eur. J. Sport Sci. 2020, 20, 981-991. [CrossRef]

31. Faul, F.; Erdfelder, E.; Buchner, A.; Lang, A.-G. Statistical power analyses using G*Power 3.1: Tests for correlation and regression analyses. Behav. Res. Methods 2009, 41, 1149-1160. [CrossRef]

32. Ministry of Culture, Sports and Tourism. Development of Standards for National Fitness in Adulthood; Ministry of Culture, Sports and Tourism: Sejong, Korea, 2010.

33. Ministry of Culture, Sports and Tourism. Improvement of National Fitness 100 Evaluation Criteria; Ministry of Culture, Sports and Tourism: Sejong, Korea, 2015.

34. Henriksen, A.; Haugen Mikalsen, M.; Woldaregay, A.Z.; Muzny, M.; Hartvigsen, G.; Hopstock, L.A.; Grimsgaard, S. Using Fitness Trackers and Smartwatches to Measure Physical Activity in Research: Analysis of Consumer Wrist-Worn Wearables. J. Med. Internet Res. 2018, 20, e110. [CrossRef] [PubMed]

35. Watson, D.; Clark, L.A. The PANAS-X: Manual for the Positive and Negative Affect Schedule-Expanded Form; University of Iowa: Iowa City, IA, USA, 1994.

36. Russell, J.A. A circumplex model of affect. J. Pers. Soc. Psychol. 1980, 39, 1161-1178. [CrossRef]

37. Russell, J.A. Core affect and the psychological construction of emotion. Psychol. Rev. 2003, 110, 145-172. [CrossRef] [PubMed]

38. Hardy, C.J.; Rejeski, W.J. Not what, but how one feels: The measurement of affect during exercise. J. Psychol Sport Exerc. 1989, 11, 304-317. [CrossRef]

39. Svebak, S.; Murgatroyd, S. Metamotivational dominance: A multimethod validation of reversal theory constructs. J. Pers. Soc. Psychol. 1985, 48, 107-116. [CrossRef]

40. Fox, S.M., III. Physical activity and the prevention of coronary heart disease. Ann. Med. Res. 1971, 3, 404-432. [CrossRef]

41. Garber, C.E.; Blissmer, B.; Deschenes, M.R.; Franklin, B.A.; Lamonte, M.J.; Lee, I.-M.; Nieman, D.C.; Swain, D.P. American College of Sports Medicine position stand. Quantity and quality of exercise for developing and maintaining cardiorespiratory, musculoskeletal, and neuromotor fitness in apparently healthy adults: Guidance for prescribing exercise. Med. Sci. Sports Exerc. 2011, 43, 1334-1359. [CrossRef] [PubMed]

42. Perez, B.; Greenwood-Robinson, M. Zumba: Ditch the Workout, Join the Party! The Zumba Weight Loss Program; Grand Central Life \& Style: New York, NY, USA, 2014.

43. Yu, J.; Park, S. K-Pop Girl Group Dance Movement Classification Systems and Visualization Implementation. Korean J. Danc. 2019, 19, 25-36.

44. Ekkekakis, P.; Petruzzello, S.J. Acute aerobic exercise and affect. Sports Med. 1999, 28, 337-347. [CrossRef]

45. Busing, K.; West, C. Determining the relationship between physical fitness, gender, and life satisfaction. SAGE Open. 2016, 6, 1-5. [CrossRef]

46. Rezazadeh, A.; Talebi, N. Relationship Between Emotion Regulation and Health-Related of Physical Fitness in Tehran Firefighters. Clin. Psychol. Personal. 2021. [CrossRef]

47. Lin, T.-W.; Kuo, Y.-M. Exercise Benefits Brain Function: The Monoamine Connection. Brain Sci. 2013, 3, 39-53. [CrossRef] [PubMed]

48. Melancon, M.; Lorrain, D.; Dionne, I. Exercise and sleep in aging: Emphasis on serotonin. Pathol. Biol. 2014, 62, 276-283. [CrossRef]

49. Klaperski, S.; von Dawans, B.; Heinrichs, M.; Fuchs, R. Effects of a 12-week endurance training program on the physiological response to psychosocial stress in men: A randomized controlled trial. J. Behav. Med. 2014, 37, 1118-1133. [CrossRef] 
50. Childs, E.; de Wit, H. Regular exercise is associated with emotional resilience to acute stress in healthy adults. Front. Physiol. 2014, 5, 161. [CrossRef] [PubMed]

51. Strahler, J.; Fuchs, R.; Nater, U.M.; Klaperski, S. Impact of physical fitness on salivary stress markers in sedentary to low-active young to middle-aged men. Psychoneuroendocrinology 2016, 68, 14-19. [CrossRef]

52. Wood, C.J.; Clow, A.; Hucklebridge, F.; Law, R.; Smyth, N. Physical fitness and prior physical activity are both associated with less cortisol secretion during psychosocial stress. Anxiety Stress Coping 2018, 31, 135-145. [CrossRef] [PubMed] 